\title{
A control strategy for controllable series capacitor in electric power systems $^{\text {is }}$
}

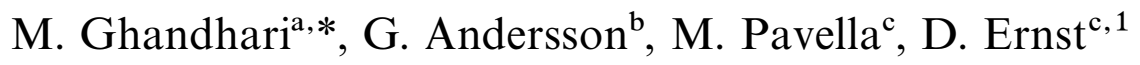 \\ ${ }^{\mathrm{a}}$ Royal Institute of Technology (KTH), Stockholm, Sweden \\ ${ }^{\mathrm{b}}$ Swiss Federal Institute of Technology, Zürich (ETH-Z), Switzerland \\ ${ }^{\mathrm{c}}$ University of Liège, Belgium
}

Received 17 August 2000; revised 25 January 2001; received in final form 14 March 2001

\begin{abstract}
It has been verified that a controllable series capacitor with a suitable control scheme can improve transient stability and help to damp electromechanical oscillations. A question of great importance is the selection of the input signals and a control strategy for this device in order to damp power oscillations in an effective and robust manner. Based on Lyapunov theory a control strategy for damping of electromechanical power oscillations in a multi-machine power system is derived. Lyapunov theory deals with dynamical systems without inputs. For this reason, it has traditionally been applied only to closed-loop control systems, that is, systems for which the input has been eliminated through the substitution of a predetermined feedback control. However, in this paper, we use Lyapunov function candidates in feedback design itself by making the Lyapunov derivative negative when choosing the control. This control strategy is called control Lyapunov function for systems with control inputs. Also, two input signals for this control strategy are used. The first one is based on local information and the second one on remote information derived by the single machine equivalent method. (C) 2001 Elsevier Science Ltd. All rights reserved.
\end{abstract}

Keywords: Power oscillations; CSC; CLF and SIME method

\section{Introduction}

Power systems exhibit various modes of oscillation due to interactions among system components. Many of the oscillations are due to synchronous generator rotors swinging relative to each other. These electromechanical oscillations (initiated by faults) which typically are in the frequency range of 0.1 to $2 \mathrm{~Hz}$, are considered in this paper.

Modern power systems are large scale and complex. Disturbances typically change the network topology and result in a nonlinear system response. Also, because of deregulation the configuration of the interconnected grid will routinely be in a state of change. Therefore, a control strategy that will counteract a wide variety of distur-

\footnotetext{
This paper was not presented at any IFAC meeting. This paper was recommended for publication in revised form by Associate Editor M. A. Johnson under the direction of Editor Mituhiko Araki

* Corresponding author. Tel.: + 46-8790-7758; fax: + 46-8790-6510.

E-mail address: mehrdad.ghandhari@ekc.kth.se (M. Ghandhari).

${ }^{1}$ Research Fellow, FNRS.
}

bances that may occur in the power system is attractive. This paper derives a control strategy for a controllable series capacitor (CSC), based on the control Lyapunov function (CLF). This control strategy is based on input signals that can easily be obtained from locally measurable variables. It has been shown in Ghandhari, Anderson and Hiskens (2000) and Ghandhari (2000) that local signals can effectively damp the electromechanical oscillations initiated by both large and small disturbances. However, a remote input signal might be more effective for this purpose. In the case of using remote input signal, an important question is which (and/or which kind of) remote information should be chosen in a multimachine power system such that the concept of the CLF is fulfilled.

For selecting a remote input signal, the single machine equivalent (SIME) method (Pavella, Ernst, \& Ruiz-Vega, 2000; Zhang, Wehenkel, Rousseaux, \& Pavella, 1997) may be a relevant choice. This method assesses the behavior of a power system in its post-fault configuration in terms of a generalized one-machine infinite bus (GOMIB) transformation to which the CLF can be applied. 
This paper is organized as follows. In Section 2 modeling of the CSC is presented. Section 3 describes the concept of CLF and its application to power systems. Section 4 gives a digest of the SIME method. We provide some numerical test results, discussions and the conclusions of this paper in Sections 5, 6 and 7, respectively.

\section{Modeling of controllable series capacitor}

A CSC can be materialized by thyristor controlled series capacitor (TCSC) and thyristor switched series capacitor (TSSC). In a simplified study CSC can be considered as a continuously controllable reactance (normally capacitance), which is connected in series with the transmission line (CIGRE Task Force 38.01.06, 1996).

Suppose a CSC is located between buses $\mathbf{i}$ and $\mathbf{j}$ in a lossless transmission line as shown in Fig. 1.

Let $x_{c}=x_{c 0}+\Delta x_{c}$, where $x_{c 0}$ is the steady-state set point and $\Delta x_{c}$ is the control modulation. Also let,

$x_{s}=x_{L}-x_{c 0}, \quad b_{s}+w=\frac{1}{x_{s}-\Delta x_{c}}, \quad b_{s}=\frac{1}{x_{s}}$.

Then, $w=u b_{s}$ and

$u=\frac{\Delta x_{c}}{x_{s}-\Delta x_{c}}$.

Thus, the real and reactive powers through the line between buses $\mathbf{i}$ and $\mathbf{j}$ are given by

$P_{i j}=b_{s} V_{i} V_{j} \sin \left(\theta_{i j}\right)+P_{s i}$,

$P_{j i}=b_{s} V_{j} V_{i} \sin \left(\theta_{j i}\right)+P_{s j}$,

$Q_{i j}=b_{s}\left(V_{i}^{2}-V_{i} V_{j} \cos \left(\theta_{i j}\right)\right)+Q_{s i}$,

$Q_{j i}=b_{s}\left(V_{j}^{2}-V_{j} V_{i} \cos \left(\theta_{j i}\right)\right)+Q_{s j}$,

where

$P_{s i}=u b_{s} V_{i} V_{j} \sin \left(\theta_{i j}\right)$,

$P_{s j}=-P_{s i}$,

$Q_{s i}=u b_{s}\left(V_{i}^{2}-V_{i} V_{j} \cos \left(\theta_{i j}\right)\right)$,

$Q_{s j}=u b_{s}\left(V_{j}^{2}-V_{j} V_{i} \cos \left(\theta_{j i}\right)\right)$.

Note that in (1), $u$ and $\Delta x_{c}$ have the same sign, since $x_{c}$ is (normally) less than $x_{L}$. If the CSC has no steady-state set point, then $x_{c 0}=0, x_{s}=x_{L}$ and $x_{c}=\Delta x_{c}$. If the CSC is not controlled, i.e. it is fixed, then $u=\Delta x_{c}=0$.

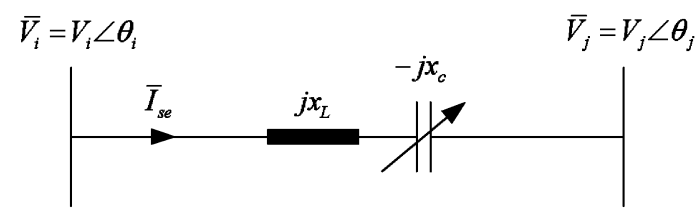

Fig. 1. A CSC in the transmission line.

\section{Control Lyapunov function}

\section{Theoretical considerations}

Power systems are most naturally described by differential algebraic (DA) models of the form $\dot{x}=f(x, y)$ and $0=g(x, y)$. The algebraic states $y$ are related to the dynamic states $x$ through the algebraic equations $g$. By virtue of the implicit function theorem, it can be shown that this model is locally equivalent to an ordinary differential equation (ODE) model

$\dot{x}=f(x, h(x))=\tilde{f}(x), \quad x \in \Omega \subseteq R^{n_{x}}$

if $\partial g / \partial y$ is nonsingular. Under certain modeling assumptions, e.g., constant admittance loads, local equivalence extends to global equivalence. This model has become known in the energy function literature as the reduced network model (RNM). The presentation of CLF in this paper is based on (2). Most ideas extend naturally to the DA model though.

Let the origin be an equilibrium point of system (2), i.e. $\tilde{f}(0)=0$, possibly after a coordinate change. A function $\mathscr{V}(x)$ is said to be a Lyapunov function for (2), if it is of class (at least) $C^{1}$ and there exists a neighborhood $Q$ of the origin such that

$\begin{array}{lllll}\mathscr{V}(x)>0 & \forall x \in Q, & x \neq 0 & \text { and } & \mathscr{V}(0)=0, \\ \dot{\mathscr{V}}(x)<0 & \forall x \in Q, & x \neq 0 & \text { and } & \dot{\mathscr{V}}(0)=0 .\end{array}$

If (2) has a Lyapunov function then the origin is locally asymptotically stable. Conversely, for any locally asymptotically stable system, a Lyapunov function exists (Khalil, 1996).

For mechanical and electrical systems, the physical energy (or energy-like) functions are often used as Lyapunov function candidates. The time derivatives of these energy functions are negative semidefinite. Therefore, these functions fail to satisfy condition (4) for Lyapunov function. However, applying La Salle's invariance principle or the theorems of Barbashin and Krasovskii (Khalil, 1996), the energy functions satisfy the asymptotic stability condition and they can be considered as Lyapunov function candidates.

Lyapunov theory deals with dynamical systems without inputs. For this reason, it has traditionally been applied only to closed-loop control systems, that is, systems for which the input has been eliminated through the substitution of a predetermined feedback control. However, some authors (Artstein, 1983; Sontag, 1989; Jurdjevic \& Quinn, 1978), started using Lyapunov function candidates in feedback design itself by making the Lyapunov derivative negative when choosing the control. Such ideas have been made precise with the introduction of the concept of a CLF for systems with control input (Freeman \& Kokotovic, 1996). 
The following discussion largely follows that in Bacciotti (1996) and references therein. Consider the control system

$\dot{x}=f(x, u), \quad x \in \Omega \subseteq R^{n_{x}}, \quad u \in R^{n_{u}}$.

We want to find conditions for the existence of a feedback control $u=u(x)$ defined in a neighborhood of the origin such that the closed-loop system $\dot{x}=f(x, u(x))$ has a locally asymptotically stable equilibrium point at the origin, i.e. $f(0, u(0))=0$. If such a function $u(x)$ exists, we say that (5) is stabilizable at the origin and the function $u(x)$ is called a stabilizing feedback law or a stabilizer. Assume that (5) is continuously stabilizable. According to the converse Lyapunov's theorem, there must be a positive definite function $\mathscr{V}(x)$ such that

$\dot{\mathscr{V}}(x)=\operatorname{grad}(\mathscr{V}) f(x, u(x))<0 \quad \forall x \in Q, \quad x \neq 0$.

A function $\mathscr{V}(x)$ satisfying (3) and (6) is called a CLF. Next, consider the affine system

$\dot{x}=f(x, u)=f_{0}(x)+\sum_{i=1}^{n_{u}} u_{i} f_{i}(x)$.

Note that $f_{0}$ and $f_{i}$ have the same dimension, i.e. $n_{x} \times 1$. For the sake of simplicity, we assume $f_{0}(0)=0$, so that we can also take $u_{i}(0)=0$. Artstein (1983) proved that there exists a stabilizer $u_{i}(x)$ for (7), if and only if (7) admits a CLF. Sontag (1989) presented explicit formulas for $u_{i}(x)$. In the case of using an energy function as a Lyapunov function candidate, the treatment of system (7) fits better in the framework of the Jurdjevic and Quinn (1978) approach. We say that (7) satisfies a Lyapunov condition of the Jurdjevic-Quinn type if there is a neighborhood $Q$ of the origin and a $C^{\infty}$ function $\mathscr{V}(x)$ such that (3) holds and $\operatorname{grad}(\mathscr{V}) f_{0}(x) \leqslant 0$ for $x \in Q$. According to the Jurdjevic and Quinn (1978) approach, a stabilizing feedback law is typically defined componentwise, setting $u(x)=\left[u_{1}(x) \ldots u_{n_{u}}(x)\right]$ and $u_{i}(x)=-\operatorname{grad}(\mathscr{V}) f_{i}(x)$, $i=1 \ldots n_{u}$. Thus, the time derivative of $\mathscr{V}(x)$ for $x \in Q$ with respect to the closed-loop system is given by

$\dot{\mathscr{V}}(x)=\operatorname{grad}(\mathscr{V}) f_{0}(x)-\sum_{i=1}^{n_{u}}\left(\operatorname{grad}(\mathscr{V}) f_{i}(x)\right)^{2} \leqslant 0$.

To summarize, just as the existence of a Lyapunov function is necessary and sufficient for the stability of a system without inputs, the existence of a CLF is necessary and sufficient for the stabilizability of a system with a control input (Freeman \& Kokotovic, 1996).

\subsection{Application to power systems}

Consider a power network which is modeled by $2 n+N$ nodes connected by lossless transmission lines which are represented by the node admittance matrix $Y=j\left[B_{k l}\right]$. The first $n$ nodes are the internal buses of the generators. The nodes $n+1$ to $2 n$ are the terminal buses of the generators where there may also be loads. Each generator terminal bus is connected with its internal bus through a lossless line with reactance equal to $x_{d}^{\prime}$, i.e. the generator transient reactance. The remaining $N$ nodes are the load buses. It is assumed that the mechanical input power of the generator is constant. The machine model considered here is the flux-decay model (the oneaxis model). Exciters and governors are not included in this model. The rest of the treatment follows that in Pai (1989) and references therein. The dynamics of the generators are described by the following differential equations(with respect to the center of inertia (COI) reference frame). For $k=1 \ldots n$,

$\dot{\tilde{\delta}}_{k}=\tilde{\omega}_{k}$,

$$
\begin{gathered}
M_{k} \tilde{\omega}_{k}=P_{m k}-P_{e k}-D_{k} \tilde{\omega}_{k}-\frac{M_{k}}{M_{T}} P_{\mathrm{COI}}, \\
T_{d o k}^{\prime} \dot{E}_{q k}^{\prime}=\frac{x_{d k}-x_{d k}^{\prime}}{x_{d k}^{\prime}} V_{k+n} \cos \left(\delta_{k}-\theta_{k+n}\right) \\
+E_{f d k}-\frac{x_{d k}}{x_{d k}^{\prime}} E_{q k}^{\prime},
\end{gathered}
$$

where $P_{\mathrm{COI}}=\sum_{k=1}^{n}\left(P_{m k}-P_{e k}\right)$ and $P_{e k}$ is the generated electrical power. Full details are given in Pai (1989).

For the lossless system, the following equations can be written at bus $k$ where $P_{k}$ is the real power and $Q_{k}$ is the reactive power injected into the system from bus $k$.

For $k=(2 n+1) \ldots(2 n+N)$

$P_{k}=\sum_{l=n+1}^{2 n+N} B_{k l} V_{k} V_{l} \sin \left(\theta_{k}-\theta_{l}\right)$,

$Q_{k}=-\sum_{l=n+1}^{2 n+N} B_{k l} V_{k} V_{l} \cos \left(\theta_{k}-\theta_{l}\right)$.

For $k=(n+1) \ldots 2 n, P_{k}$ and $Q_{k}$ are similar, but also take account of generated real and reactive power (Pai, 1989).

Real load at each bus is represented by a constant load and reactive load by an arbitrary function of voltage at the respective bus.

Thus, for $k=(n+1) \ldots(2 n+N)$

$P_{L k}=P_{L k}^{0}, \quad Q_{L k}=f_{q k}\left(V_{k}\right)$.

Therefore, for $k=(n+1) \ldots(2 n+N)$ the power flow equations can be written as

$P_{k}+P_{L k}=0$,

$Q_{k}+Q_{L k}=0$.

An energy function for the differential algebraic equations (9) and (10) is given by

$\mathscr{V}\left(\tilde{\omega}, \tilde{\delta}, E_{q}^{\prime}, V, \tilde{\theta}\right)=\mathscr{V}_{1}+\sum_{k=1}^{8} \mathscr{V}_{2 k}+C_{0}$, 
where

$$
\begin{aligned}
& \mathscr{V}_{1}=\frac{1}{2} \sum_{k=1}^{n} M_{k} \tilde{\omega}_{k}^{2} \\
& \mathscr{V}_{21}=-\sum_{k=1}^{n} P_{m k} \tilde{\delta}_{k}, \\
& \mathscr{V}_{22}=\sum_{k=n+1}^{2 n+N} P_{L k} \widetilde{\theta_{k}}, \\
& \mathscr{V}_{23}=\sum_{k=n+1}^{2 n+N} \int \frac{Q_{L k}}{V_{k}} \mathrm{~d} V_{k} \text {, } \\
& \mathscr{V}_{24}=\sum_{k=n+1}^{2 n} \frac{1}{2 x_{d k-n}^{\prime}}\left[E_{q k-n}^{\prime 2}+V_{k}^{2}\right. \\
& \left.-2 E_{q k-n}^{\prime} V_{k} \cos \left(\delta_{k-n}-\theta_{k}\right)\right], \\
& \mathscr{V}_{25}=-\frac{1}{2} \sum_{k=n+1}^{2 n+N} \sum_{l=n+1}^{2 n+N} B_{k l} V_{k} V_{l} \cos \left(\theta_{k}-\theta_{l}\right) \text {, } \\
& \mathscr{V}_{26}=\sum_{k=n+1}^{2 n} \frac{x_{d k-n}^{\prime}-x_{q k-n}}{4 x_{d k-n}^{\prime} x_{q k-n}} \\
& {\left[V_{k}^{2}-V_{k}^{2} \cos \left(2\left(\delta_{k-n}-\theta_{k}\right)\right)\right],} \\
& \mathscr{V}_{27}=-\sum_{k=1}^{n} \frac{E_{f d k} E_{q k}^{\prime}}{x_{d k}-x_{d k}^{\prime}}, \\
& \mathscr{V}_{28}=\sum_{k=1}^{n} \frac{E_{q k}^{\prime 2}}{2\left(x_{d k}-x_{d k}^{\prime}\right)} \text {. }
\end{aligned}
$$

$\mathscr{V}_{1}$ is known as the kinetic energy and $\sum \mathscr{V}_{2 k}$ as the potential energy. $C_{0}$ is a constant such that at the postfault stable equilibrium point, the energy function is zero.

Using the notation $[\mathrm{d} \mathscr{V} / \mathrm{d} t]_{\tilde{\omega}}$ for $\partial \mathscr{V} / \partial \tilde{\omega} \mathrm{d} \tilde{\omega} / \mathrm{d} t$, and similarly for the other states, we have

$$
\begin{aligned}
& {\left[\frac{\mathrm{d} \mathscr{V}_{1}}{\mathrm{~d} t}\right]_{\tilde{\omega}}+\left[\frac{\mathrm{d} \mathscr{V}_{21}}{\mathrm{~d} t}+\frac{\mathrm{d} \mathscr{V}_{24}}{\mathrm{~d} t}+\frac{\mathrm{d} \mathscr{V}_{26}}{\mathrm{~d} t}\right]_{\tilde{\delta}}} \\
& =-\sum_{k=1}^{n} D_{k}\left(\tilde{\omega}_{k}\right)^{2} \\
& {\left[\frac{\mathrm{d} \mathscr{V}_{22}}{\mathrm{~d} t}+\frac{\mathrm{d} \mathscr{V}_{24}}{\mathrm{~d} t}+\frac{\mathrm{d} \mathscr{V}_{25}}{\mathrm{~d} t}+\frac{\mathrm{d} \mathscr{V}_{26}}{\mathrm{~d} t}\right]_{\tilde{\theta}}} \\
& \quad=\sum\left(P_{k}+P_{L k}\right) \tilde{\theta}_{k}=0, \\
& \left.\frac{\mathrm{d} \mathscr{V}_{23}}{\mathrm{~d} t}+\frac{\mathrm{d} \mathscr{V}_{24}}{\mathrm{~d} t}+\frac{\mathrm{d} \mathscr{V}_{25}}{\mathrm{~d} t}+\frac{\mathrm{d} \mathscr{V}_{26}}{\mathrm{~d} t}\right]_{V} \\
& =\sum\left(Q_{k}+Q_{L k}\right) \frac{\dot{V}_{k}}{V_{k}}=0, \\
& {\left[\frac{\mathrm{d} \mathscr{V}_{27}}{\mathrm{~d} t}+\frac{\mathrm{d} \mathscr{V}_{28}}{\mathrm{~d} t}+\frac{\mathrm{d} \mathscr{V}_{24}}{\mathrm{~d} t}\right]_{E_{q}^{\prime}}=-\sum_{k=1}^{n} \frac{T_{d o k}^{\prime}}{x_{d k}-x_{d k}^{\prime}}\left(\dot{E}_{q k}^{\prime}\right)^{2}}
\end{aligned}
$$

Thus, the time derivative of the energy function is

$$
\begin{aligned}
\frac{\mathrm{d} \mathscr{V}}{\mathrm{d} t} & =-\sum_{k=1}^{n} D_{k}\left(\tilde{\omega}_{k}\right)^{2}-\sum_{k=1}^{n} \frac{T^{\prime}{ }_{d o k}}{x_{d k}-x_{d k}^{\prime}}\left(\dot{E}_{q k}^{\prime}\right)^{2} \\
& =\dot{\mathscr{V}}_{\text {NOCSC }} \leqslant 0 .
\end{aligned}
$$

Now assume that a CSC is located between buses $\mathbf{i}$ and j in the transmission system. The introduction of the CSC does not alter the energy function (11). However, it does alter $\dot{\mathscr{V}}$; in particular the terms (13) and (14) no longer sum to zero. To see this, consider the $i$ th term of (13), i.e. $\left(P_{i}+P_{L i}\right) \dot{\widetilde{\theta}}_{i}$. Without a CSC connected to bus $\mathbf{i}$, $\left(P_{i}+P_{L i}\right)=0$, resulting in the zero summation of (13). However, when the CSC is connected, power balance gives $\left(P_{i}+P_{L i}+P_{s i}\right)=0$. Therefore, with the CSC connected, the ith term of (13) becomes $\left(P_{i}+P_{L i}\right) \tilde{\theta}_{i}=-P_{s i} \tilde{\theta}_{i}$. A similar argument follows for the $j$ th term of (13) and the corresponding terms of (14), resulting in

$$
\begin{aligned}
& {\left[\frac{\mathrm{d} \mathscr{V}_{22}}{\mathrm{~d} t}+\frac{\mathrm{d} \mathscr{V}_{24}}{\mathrm{~d} t}+\frac{\mathrm{d} \mathscr{V}_{25}}{\mathrm{~d} t}+\frac{\mathrm{d} \mathscr{V}_{26}}{\mathrm{~d} t}\right]_{\tilde{\theta}}} \\
& =-P_{s i} \dot{\tilde{\theta}}_{i}-P_{s j} \dot{\tilde{\theta}}_{j}, \\
& =-P_{s i} \tilde{\theta}_{i}-P_{s j} \tilde{\theta}_{j}, \\
& {\left[\frac{\mathrm{d} \mathscr{V}_{22}}{\mathrm{~d} t}+\frac{\mathrm{d} \mathscr{V}_{24}}{\mathrm{~d} t}+\frac{\mathrm{d} \mathscr{V}_{25}}{\mathrm{~d} t}+\frac{\mathrm{d} \mathscr{V}_{26}}{\mathrm{~d} t}\right]_{V}} \\
& =-Q_{s i} \frac{\dot{V}_{i}}{V_{i}}-Q_{s j} \frac{\dot{V}_{j}}{V_{j}}
\end{aligned}
$$

Note that (12) and (15) are unaffected by the introduction of a CSC.

Therefore, the time derivative of the energy function becomes

$\frac{\mathrm{d} \mathscr{V}}{\mathrm{d} t}=\dot{\mathscr{V}}_{\mathrm{NOCSC}}+\dot{\mathscr{V}}_{\mathrm{CSC}} \leqslant \dot{\mathscr{V}}_{\mathrm{CSC}}$

where

$$
\begin{aligned}
\dot{V}_{\mathrm{CSC}} & =-\frac{1}{2} b_{s} u \frac{\mathrm{d}}{\mathrm{d} t}\left[V_{i}^{2}+V_{j}^{2}-2 V_{i} V_{j} \cos \left(\theta_{i j}\right)\right] \\
& =-\frac{1}{2} b_{s} u \frac{\mathrm{d}}{\mathrm{d} t}\left|\bar{V}_{i j}\right|^{2}=-\frac{1}{2} b_{s} u \frac{\mathrm{d}}{\mathrm{d} t}\left[x_{L} I_{\mathrm{csc}}-V_{\mathrm{csc}}\right]^{2}
\end{aligned}
$$

The energy function will be a CLF, if $\dot{\mathscr{V}}_{\mathrm{CSC}}$ is negative. Therefore, the following control law is suggested:

$u=k_{\text {local }} \frac{\mathrm{d}}{\mathrm{d} t}\left[x_{L} I_{\mathrm{csc}}-V_{\mathrm{csc}}\right]^{2}$,

where $I_{\text {csc }}$ is the absolute value of current through CSC, $V_{\text {csc }}$ is the absolute value of voltage over CSC and $k_{\text {local }}$ is a positive gain which is chosen individually to obtain appropriate damping. Thus, the control law based on the 
CLF relies only on locally measurable information and is independent of system topology and modeling of power system components. Also, this control law does not require knowledge of the post-fault stable equilibrium point.

\section{Single machine equivalent}

In the case of using remote input signal, an important question is which (and/or which kind of) remote information should be chosen in a multi-machine power system such that the concept of the CLF is fulfilled.

For selecting a remote input signal, the SIME method (Pavella et al., 2000; Zhang et al., 1997) may be a relevant choice. This method assesses the behavior of a power system in its post-fault configuration in terms of a generalized one-machine infinite bus (GOMIB) transformation to which the CLF can be applied.

\subsection{Foundations}

SIME is a hybrid direct-temporal transient stability method, which transforms the trajectories of a multimachine power system into the trajectory of a GOMIB system, whose parameters are time-varying (Pavella et al., 2000).

The GOMIB parameters are its rotor angle $(\delta)$, rotor speed $(\omega)$, inertia coefficient $(M)$, mechanical power $\left(P_{\mathrm{m}}\right)$, and electrical power $\left(P_{\mathrm{e}}\right)$.

Basically, SIME deals with the post-fault configuration of a power system subjected to a disturbance which presumably drives it to instability. However, the GOMIB is also valid on a borderline stable case. Under such conditions, SIME uses a time-domain program in order to identify the mode of separation of its machines into two groups, namely critical (subscript $\mathrm{C}$ ) and non-critical machines (subscript NC) which are replaced successively by a two-machine equivalent. Then, this two-machine equivalent is replaced by a GOMIB system. By definition, the critical machines are the machines responsible for the loss of synchronism. Let

$$
M_{\mathrm{C}}=\sum_{i \in \mathrm{C}} M_{i}, \quad M_{\mathrm{NC}}=\sum_{j \in \mathrm{NC}} M_{j}, \quad M_{T}=M_{\mathrm{C}}+M_{\mathrm{NC}} .
$$

The angle and the speed of the GOMIB system are expressed by

$$
\begin{aligned}
& \delta_{\mathrm{GOMIB}}=\delta_{\mathrm{C}}-\delta_{\mathrm{NC}}, \\
& \omega_{\mathrm{GOMIB}}=\omega_{\mathrm{C}}-\omega_{\mathrm{NC}},
\end{aligned}
$$

where

$\delta_{\mathrm{C}}=M_{\mathrm{C}}^{-1} \sum_{i \in \mathrm{C}} M_{i} \delta_{i}, \quad \delta_{\mathrm{NC}}=M_{\mathrm{NC}}^{-1} \sum_{j \in \mathrm{NC}} M_{j} \delta_{j}$,
$\omega_{\mathrm{C}}=M_{\mathrm{C}}^{-1} \sum_{i \in \mathrm{C}} M_{i} \omega_{i}, \quad \omega_{\mathrm{NC}}=M_{\mathrm{NC}}^{-1} \sum_{j \in \mathrm{NC}} M_{j} \omega_{j}$.

By refreshing the GOMIB parameters at each integration time-step, SIME provides a faithful replica of the transient stability assessment of the multi-machine system, and also additional interesting pieces of information, such as stability margins, identification of the mode of instability and corresponding critical machines, sensitivity analysis and control techniques (Zhang et al., 1997).

\subsection{Control law based on SIME}

Consider the one-machine infinite bus (OMIB) system shown in Fig. 2 in which $x$ includes line reactance, transformer reactance and generator transient reactance.

Dynamics of this system are given by

$\dot{\delta}=\omega$,

$\dot{\omega}=\left[P_{\mathrm{m}}-P_{\mathrm{e}}-D \omega\right] / M$,

where $P_{\mathrm{e}}=P_{\max } \sin (\delta), P_{\max }=b E^{\prime} V$ and $b=1 / x . P_{\mathrm{m}}$ is the mechanical power which is constant and $D$ is a positive damping constant. Let $x=\left[\begin{array}{ll}\delta & \omega\end{array}\right]^{\mathrm{T}}$ and consider (22) as $\dot{x}=f_{0}(x)$. For (22) the following energy function exists:

$\mathscr{V}=0.5 M \omega^{2}-P_{\mathrm{m}} \delta-P_{\max } \cos (\delta)+C_{0}$.

The time derivative of (23) along the trajectory of (22) is given by

$\dot{\mathscr{V}}=\operatorname{grad}(\mathscr{V}) \cdot f_{0}(x)=-D \omega^{2} \leqslant 0$.

Thus, the energy function (23) is a Lyapunov function candidate for (22). Having a CSC in the system (22), then $P_{\mathrm{e}}=(1+u) P_{\max } \sin (\delta)$. Thus, the following dynamics are given

$\dot{x}=f_{0}(x)+u f_{1}(x)$,

where $f_{1}(x)=\left[0-c_{1} \sin (\delta)\right]^{\mathrm{T}}$ and $c_{1}=P_{\max } / M$.

Using (23) as a Lyapunov function candidate for system (25), we have

$\dot{\mathscr{V}}=\operatorname{grad}(\mathscr{V})\left(f_{0}(x)+u f_{1}(x)\right) \leqslant u \operatorname{grad}(\mathscr{V}) f_{1}(x)$.

By virtue of the Jurdjevic and Quinn (1978) approach, the following stabilizing control law is given for CSC, $(k>0)$ :

$u=-\operatorname{grad}(\mathscr{V}) f_{1}(x)=k \sin (\delta) \omega$,

which makes (26) negative. Therefore, the energy function (23) becomes a CLF for the system (25).

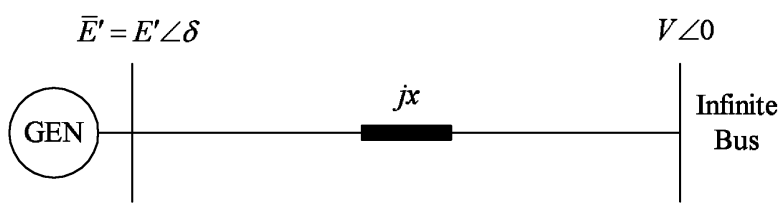

Fig. 2. The OMIB system. 


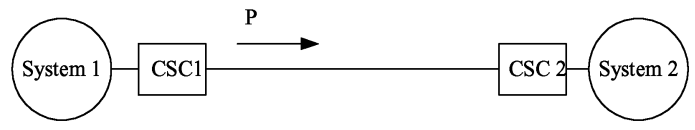

Fig. 3. Brazilian North-South interconnection.

Now, consider the power system shown in Fig. 3 without CSCs. After a large disturbance, based on the SIME method, the post-fault dynamics of the corresponding GOMIB system are given by

$\dot{\delta}_{\mathrm{GOMIB}}=\omega_{\mathrm{GOMIB}}$,

$\dot{\omega}_{\mathrm{GOMIB}}=M_{T}^{-1}\left[P_{\mathrm{m}_{\mathrm{GOM}}}-P_{\mathrm{e}_{\mathrm{GOM}}}\right]$,

where $P_{\mathrm{m}_{\text {Gomis }}}$ is the equivalent mechanical input power and $P_{\mathrm{e}_{\text {сом⿰口口 }}}$ is the equivalent electrical output power calculated by the time simulation program. They can be defined by

$P_{\mathrm{m}_{\mathrm{GoMm}}}=M_{T}^{-1}\left[M_{\mathrm{NC}} \sum_{i \in \mathrm{C}} P_{\mathrm{m} i}-M_{\mathrm{C}} \sum_{j \in \mathrm{NC}} P_{\mathrm{m} j}\right]$

and

$P_{\mathrm{e}_{\mathrm{GOM}}}=\alpha \sin \left(\beta \delta_{\mathrm{GOMIB}}+\gamma\right)$,

where parameters $\alpha, \beta$ and $\gamma$ are time-varying, and are calculated at each integration time-step.

Within the stability region of a real power system, (29) and (30) are indeed bounded. Therefore, it is reasonable to assume that there exist a constant $P_{\mathrm{m}_{\mathrm{app}}}$ and a constant $P_{\text {max }_{\text {app }}}$ such that

$\left\|P_{\mathrm{m}_{\mathrm{GOM} I \mathrm{~B}}}-P_{\mathrm{m}_{\mathrm{app}}}\right\|$

and

$\left\|P_{\mathrm{e}_{\text {Gоммів }}}-P_{\mathrm{e}_{\text {app }}}\right\|$

are small, where $P_{\mathrm{e}_{\mathrm{app}}}=P_{\text {max }_{\mathrm{app}}} \sin \left(\delta_{\mathrm{GOMIB}}\right)$.

Now, the right-hand side of (28) is simplified by

$\dot{\delta}_{\mathrm{GOMIB}}=\omega_{\mathrm{GOMIB}}$,

$\dot{\omega}_{\mathrm{GOMIB}}=M_{T}^{-1}\left[P_{\mathrm{m}_{\text {app }}}-P_{\text {max }_{\mathrm{app}}} \sin \left(\delta_{\mathrm{GOMIB}}\right)\right]$.

Let $x=\left[\begin{array}{ll}\delta_{\mathrm{GOMIB}} & \omega_{\mathrm{GOMIB}}\end{array}\right]^{\mathrm{T}}$. Let also $F(x)$ and $f_{0}(x)$ denote the right-hand sides of (28) and (31), respectively. System (28) can now be rewritten as

$$
\begin{aligned}
\dot{x} & =F(x) \\
& =f_{0}(x)+\left[F(x)-f_{0}(x)\right] \\
& =f_{0}(x)+p(x) .
\end{aligned}
$$

Assuming $p(x)=0$, the GOMIB system has the same dynamics as the physical OMIB system, i.e. system (22) with $D=0$. Consequently, a similar Lyapunov function to (23) (in which $M, P_{\mathrm{m}}, P_{\max }, \omega$ and $\delta$ are replaced by $M_{T}, P_{\mathrm{m}_{\text {app }}}, P_{\text {max }_{\text {app }}}, \omega_{\mathrm{GOMIB}}$ and $\delta_{\mathrm{GOMIB}}$, respectively) can be used for the GOMIB system.
Having a CSC between the two systems in Fig. 3, the dynamics of the GOMIB system with the CSC is similar to the dynamics of the physical OMIB system with the CSC, i.e. system (25). Thus, the control strategy for the CSC in the GOMIB system is similar to (27), that is,

$u=k_{\text {remote }} \sin \left(\delta_{\mathrm{GOMIB}}\right) \omega_{\mathrm{GOMIB}}$,

where $k_{\text {remote }}$ is a positive gain. Mathematically, any positive $k_{\text {local }}$ and $k_{\text {remote }}$ should stabilize the system. In practice, there are however limitations for these gains, see Section 8.4 in Ghandhari (2000).

\section{Numerical example}

The control laws (20) and (33) have been applied to various test systems, see Ghandhari (2000). In this section, the Brazilian power system is used for applying the control laws (20) and (33). Note that these control laws were developed assuming some modeling restrictions. However, they will be applied to a real system that is not subject to those modeling restrictions. Fig. 3 shows a sketch of the Brazilian North-South interconnection described in Carraro and Salomao (1999). The North/Northeast interconnected system (i.e. System 1 in Fig. 3) consists of large hydro-generating complexes that are linked to 230 and $500 \mathrm{kV}$ transmission networks. The South/Southeast/Midwest interconnected system (i.e. System 2 in Fig. 3) consists of a large number of hydrogenerating plants linked to the main load centers by transmission networks operating at 138 and $750 \mathrm{kV}$. The North-South (i.e. System 1-System 2) interconnect transmission line is $1028 \mathrm{~km}$ with a circuit rating of $1300 \mathrm{MW}$. Two CSCs have been located in the North-South interconnect transmission line, full details are given in Carraro and Salomao (1999). The CSC location was recommended for reasons such as availability of reliable auxiliary services, support for operation and maintenance and further expansion of the transmission system. For both CSCs, the steady-state set points (i.e. $x_{c 0}$ ) are $15.84 \Omega$; and $13.2 \leqslant x_{c} \leqslant 40 \Omega$.

Four cases are studied. In case 1 and case 2, a line and a shunt are tripped in System 1 and System 2, respectively, after a fault. In both cases, the fault is cleared after $100 \mathrm{~ms}$. In case 3 , a generator with a production of $606 \mathrm{MW}$ is disconnected in System 1. In case 4, a generator with a production of $395 \mathrm{MW}$ is disconnected in System 2.

Fig. 4 shows the variation of $P$ (identified in Fig. 3) and the variation of the $x_{c}$ of the first CSC, i.e. CSC1 in Fig. 3, vs. time when the CSCs are not controlled and controlled by (20) and (33), respectively. The simulation results show the ability of the control laws to damp power system oscillations for various cases. They also show that the proposed system has better damping with control law (33) than control law (20) for the proposed cases. 

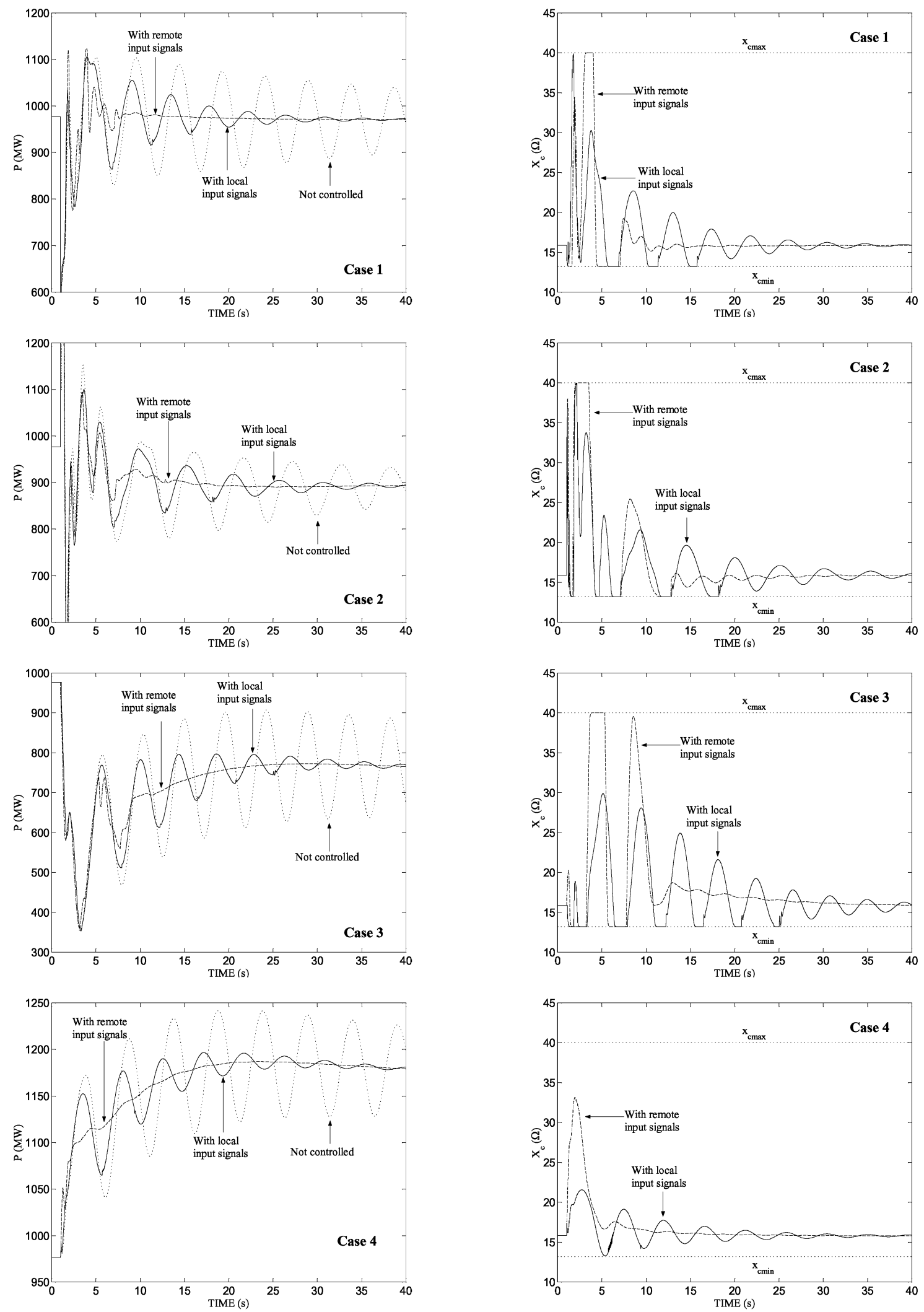

Fig. 4. Variation of $P$ and the $x_{c}$ of CSC1 vs. time in the Brazilian network. 

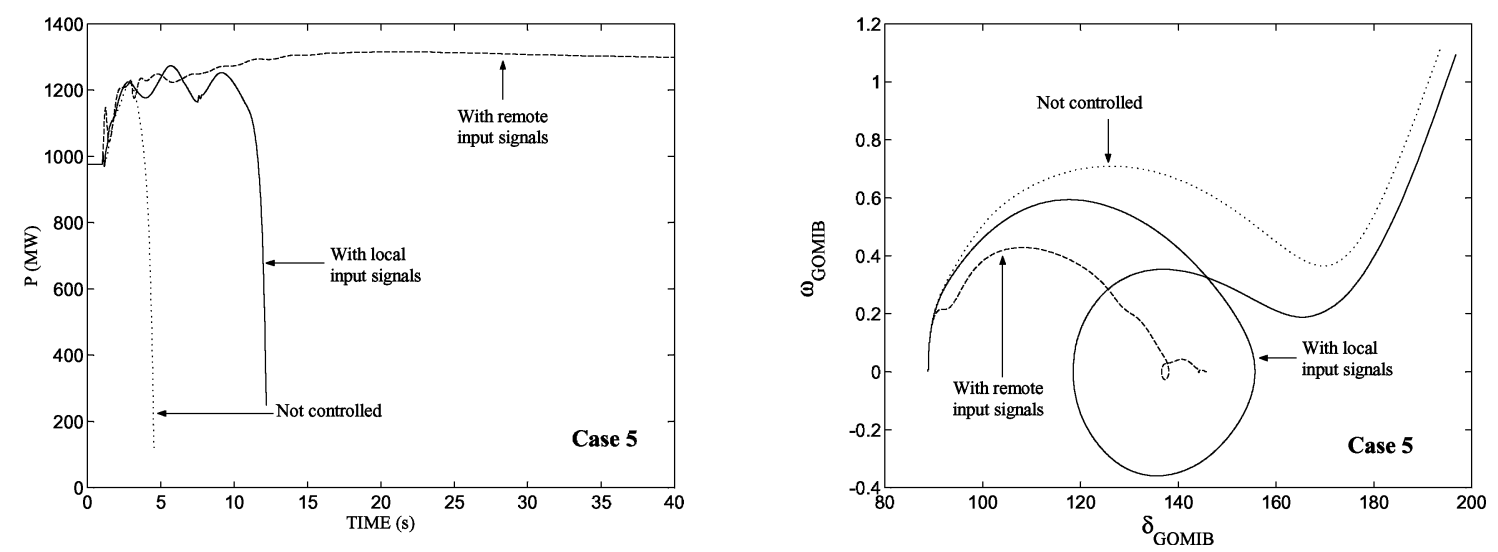

Fig. 5. Variation of $P$ and phase portrait of the corresponding GOMIB for case 5.

Next, an extreme case (case 5) is studied. In this case, a generator with a production of $800(\mathrm{MW})$ is disconnected in the South/Southeast/Midwest. This case implies that the power through the North-South interconnect transmission line (in the post-fault steady state) is almost $1300(\mathrm{MW})$ which is the circuit rating of the North-South interconnect transmission line. Fig. 5 shows that the control law based on the local input signals cannot stabilize the system for this case. However, the system is stabilized when the remote input signals are used. Note, however, that both control laws are based on the CLF. They have also been derived based on a simplified system.

\section{Discussion and future work}

The model used in the development of the control law (20) had a very specific form. It was convenient for obtaining a Lyapunov function, but it only approximately describes actual power system behavior. The issue of modeling approximations, and their influence on the stabilization of power systems, is yet to be fully addressed. Model (2) will be used to illustrate these ideas.

Assume that the model used in the development of the CLF has the form

$\dot{x}=f_{0}(x)$.

For instance, $f_{0}(x)$ can be considered as the ODE model, (by virtue of the implicit function theorem), of the differential algebraic equations (9) and (10) in which the power system is assumed to be lossless, the real loads are constant and also, one-axis model without turbine regulator and AVR are used for the generators. Assume also that the actual system is described by $\dot{x}=F(x)$, that is, the power system is not subjected to the above modeling restrictions, see also systems (28), (31) and(32). Simple manipulation gives

$\dot{x}=F(x)=F(x)+f_{0}(x)-f_{0}(x)=f_{0}(x)+p(x)$.
Since it is difficult to find a Lyapunov function for (35), a Lyapunov function is derived for (34), i.e. when $p(x)=0$, and a control law is established which makes that Lyapunov function a CLF. The following questions arise. How does this control law, derived for (34), affect $p(x)$ ? In the context of CSC control, how good is the control law (20) when the system is lossy, and more detailed models are used for generators and loads? More precisely, how good are the control laws (20) and (33) when $p(x) \neq 0$ ?

The simulation results in this paper provide a partial answer. They indicate that the control laws are not sensitive to the model approximations. However, it is important to obtain an analytical justification of this observation. The following theorems (see Krasovskii and Brenner (1963) Chapter 5) may provide a partial answer.

We present the analysis in terms of the zero equilibrium point. The stability of any other equilibrium point can be obtained by simply translating the coordinate system so that the equilibrium of interest is at the origin in the new coordinates. Let the origin be an equilibrium point for the system (34). Let also $\mathscr{X}(t)$ denote the solution of the actual system (35). We say that the origin is totally stable, if for every positive number $\varepsilon$, however small, there are two positive numbers $\eta_{1}(\varepsilon)$ and $\eta_{2}(\varepsilon)$ such that

$\|\mathscr{X}(t)\|<\varepsilon$ for $t>0$,

provided that

$\|\mathscr{X}(0)\|<\eta_{1}(\varepsilon)$

and that in the domain $\|x\|<\varepsilon$ and $t>0$, the inequality

$\|p(x)\|<\eta_{2}(\varepsilon)$

is satisfied.

Note that in the definition of the total stability, it is not required that $p(x)$ should be zero at the origin, i.e. $p(0) \neq 0$. The only requirement on the function $p(x)$ is that this function is bounded in modulus for sufficiently small values of $x$, i.e. $p(x)$ remains small for all $t>0$. 
Theorem 1. If the origin of the system (34) is asymptotically stable, it is also totally stable.

Applying the converse Lyapunov theorem, we can then state the following theorem.

\section{Theorem 2. If there exists a Lyapunov function for system} (34), the origin is totally stable.

The requirement that the function $p(x)$ be small for all $t>0$ is not realistic. It is more realistic to require that $p(x)$ may assume large values in certain small intervals of time while being small most of the time. A function $p(x)$ with this property is called bounded in the mean. It can be shown that Theorem 2 is also valid for $p(x)$ that is bounded in the mean (Krasovskii \& Brenner, 1963).

The control law (20) relies only on locally measurable information. Thus, the input signals are inexpensive, fast and reliable. This control law has been applied to various test systems for small and large disturbances as well as meshed and radial systems. The simulation results (see e.g. Ghandhari et al. (2000) and Ghandhari (2000), have shown that (20) is an effective and robust control law. The control law (33) is new. It relies on remote input signals, i.e. data from all machines in the system. Thus, the input signals can be expensive and less reliable in a real power system. However, due to the latest development in signal and communication technology, these issues may be solved in the near future. The simulation results in this paper have shown that the proposed system has better damping with the control law (33) than the control law (20) for the proposed cases. However, the following questions arise. How effective is this control law for small disturbances? How does it work in a meshed system in which several CSCs are located in different locations? How fast can SIME predict on-line a GOMIB system for large as well as small disturbances? Answers to these questions are the basis for a project which will be performed in a collaboration between the Royal Institute of Technology in Stockholm and the University of Liège.

\section{Conclusions}

It has been shown that the controllable series capacitor (CSC) provides an effective means of adding damping to power systems.

Two control laws, i.e. the control laws (20) and (33), have been derived for CSC based on control Lyapunov function (CLF) concepts. These control laws do not require knowledge of the post-fault stable equilibrium points. The control law (20) relies only on locally measurable information; but the control law (33) relies on remote input signals, i.e. data from all machines in the system. The simulation results have shown that the pro- posed system has better damping with the control law (33) than the control law (20) for the proposed cases. However, further research is required for a general conclusion regarding the control law (33).

\section{References}

Artstein, Z. (1983). Stabilization with relaxed controls. Nonlinear Analysis, Theory, Methods and Applications, 7(11), 1163-1173.

Bacciotti, A. (1996). Local stabilizability of nonlinear control systems. Singapore: World Scientific Publishing Co. Pte. Ltd.

Carraro, B., \& Salomao, J. (1999). Power corridor unites Brazil's network. Transmission and Distribution World, 70-74.

CIGRE Task Force 38.01.06, . (1996). Load flow control in high voltage systems using FACTS controllers. CIGRE Technical brochure 51.

Freeman, R. A., \& Kokotovic, P. V. (1996). Robust nonlinear control design. Basel: Birkhäuser.

Ghandhari, M. (2000). Control Lyapunov functions: A control strategy for damping of power oscillations in large power systems. Ph.D. thesis, Royal Institute of Technology, TRITA-EES-0004, ISSN 1100-1607, http://media.lib.kth.se:8080/dissengrefhit.asp? dissnr $=3039$.

Ghandhari, M., Andersson, G., \& Hiskens, I. A. (2000). Control Lyapunov functions for controllable series devices. SEPOPE, Brazil, (invited paper), May.

Jurdjevic, V., \& Quinn, J. P. (1978). Controllability and stability. Journal of Differential Equations, 28, 381-389.

Khalil, H. K. (1996). Nonlinear systems (2nd ed.). Englewood Cliffs, NJ: Prentice-Hall, Inc.

Krasovskii, N. N., \& Brenner, J. L. (1963). Stability of motion. Stanford, California: Stanford University Press.

Pai, M. A. (1989). Energy function analysis for power system stability. Dordrecht: Kluwer Academic Publishers.

Pavella, M., Ernst, D., \& Ruiz-Vega, D. (2000). Power system transient stability analysis and control. Dordrecht: Kluwer Academic Publishers.

Sontag, E. (1989). A universal construction of Artstein's theorem on nonlinear stabilization. Systems and Control Letters, 13, 117-123.

Zhang, Y., Wehenkel, L., Rousseaux, P., \& Pavella, M. (1997). SIME: A hybrid approach to fast transient stability assessment and contingency selection. EPES, 19(3), 195-208.

Mehrdad Ghandhari received his M.Sc., Tech.Lic and Ph.D. degrees in electrical engineering from Royal Institute of Technology, Sweden in 1995, 1997 and 2000, respectively. He is currently a Postdoctoral Fellow at the Royal Institute of Technology (KTH), Stockholm, Sweden.

Göran Andersson received his M.Sc. and Ph.D. degrees from the University of Lund in 1975 and 1980, respectively. In 1980, he joined ASEA:s HVDC-division and in 1986 he was appointed professor in Electric Power Systems at the Royal Institute of Technology (KTH), Stockholm, Sweden. Since 2000 he is a Professor at the Swiss Federal Institute of Technology, Zürich (ETH-Z), Switzerland.

Damien Ernst received the Electromechanical engineering degree in 1998 from the University of Liège where he is currently a Ph.D. student. His research is in the area of transient stability assessment and control.

Mania Pavella received the Electrical (Electronics) engineering degree and the Ph.D. degree from the University of Liège, where she is presently a Professor. Her research interests lie in the field of the electric power system analysis and control. 